hep-th/0402187

\title{
The $(2,0)$ supersymmetric theory of tensor multiplets and self-dual strings in six dimensions
}

\author{
Pär Arvidsson ${ }^{1}$, Erik Flink ${ }^{2}$ and Måns Henningson ${ }^{3}$ \\ Department of Theoretical Physics \\ Chalmers University of Technology and Göteborg University \\ SE-412 96 Göteborg, Sweden
}

\begin{abstract}
We construct a unique $(2,0)$ supersymmetric action in six dimensions, describing a tensor multiplet interacting with a self-dual string. It is a sum of four terms: A free kinetic term for the tensor multiplet fields integrated over Minkowski space, a Nambu-Goto type kinetic term for the string integrated over the string worldsheet, a Wess-Zumino type electromagnetic coupling integrated over the world-volume of a Dirac membrane attached to the string, and a direct interaction of such Dirac membranes. In addition to supersymmetry, the action is also invariant under a local symmetry, which allows us to choose the Dirac membrane world-volume freely and eliminate half of the fermionic degrees of freedom on the string world-sheet.
\end{abstract}

\footnotetext{
${ }^{1}$ par@fy.chalmers.se

2 erik.flink@fy.chalmers.se

${ }^{3}$ mans@fy.chalmers.se
} 


\section{Introduction}

The six-dimensional $(2,0)$ theories are one of the most remarkable and surprising discoveries in string theory during the past decade [1]. The existence of these theories can be inferred by studying string theory or $M$-theory on a ten- or eleven-dimensional space-time which contains a six-dimensional impurity. Under certain conditions, degrees of freedom supported at the locus of this impurity then decouple from the remaining bulk degrees of freedom and could be described by a separate six-dimensional quantum theory. However, an intrinsically six-dimensional definition of these theories is not yet known, and finding such a description appears as a major goal of our work. At first sight, it then appears natural to work at the origin of the moduli space, but here a $(2,0)$ theory is described by a strongly coupled conformal field theory, which is hard to understand with current methods. In previous papers [2, we have instead followed an approach based on working at a generic point in the moduli space, where conformal invariance is spontaneously broken, and the theory can be thought of as describing the dynamics of massless fields coupled to tensile strings.

In this paper, we will construct an interacting theory of such massless fields and tensile strings. We will limit ourselves to the simplest $A_{1}$ version of $(2,0)$ theory, where there is a single species of fields and a single species of strings. Although we have taken steps in this direction in our previous publications, we have strived to make the present paper self-contained. In the next section, we will review the free kinetic terms for the fields in six-dimensional Minkowski space. The main difficulty here is that in order to give a Lagrangian description, we must include additional degrees of freedom that are not really part of $(2,0)$ theory [3]. This is consistent though, provided that the interactions that we introduce later are constructed so that these extra degrees of freedom remain decoupled. In section three, we will discuss the corresponding on-shell superspace [4]. Although this is really only appropriate for the free theory, it will still be an important tool for constructing the interactions. In section four, we couple a string to a prescribed background of space-time fields that fulfill the free equations of motion. There are two interaction terms: One is a NambuGoto type term for the string, with the tension given by a space-time scalar field. The other is a Wess-Zumino type term that encodes the electromagnetic coupling of the string to a space-time tensor field. The Wess-Zumino term is constructed using a Dirac membrane, the boundary of which is given by the string. In addition to being supersymmetric, the sum of these two terms is invariant under a local symmetry, which has two important consequences. First of all it allows us to choose the Dirac membrane freely, so that only the actual string is of physical significance [5]. Furthermore, this symmetry decouples half of the fermionic variables on the string world-sheet, reflecting the BPS-saturated property of the string [6]. In the last section we will combine the Minkowski space kinetic terms with the Nambu-Goto and Wess-Zumino terms. To ensure that the complete action still is invariant under supersymmetry and the local symmetry, we have to modify the transformation laws of the space-time fields with additional terms. We also have to add an additional term to the action describing 
direct Dirac membrane interactions. The exact description of this term is somewhat implicit, but we also show how its purely bosonic part can be constructed completely explicitly.

The final action that we have constructed is unique, with no adjustable parameters. This seems to indicate that the resulting quantum theory, when properly defined, may have some finiteness property. We hope to be able to investigate these questions in forthcoming publications.

\section{The tensor multiplet term}

We will begin by explaining our notation for $(2,0)$ supersymmetry in six-dimensional Minkowski space $M$. This symmetry of course contains the Lorentz group $S O(5,1)$. We denote chiral and anti chiral Lorentz spinors by a subscript or superscript index $\alpha, \beta, \ldots=1,2,3,4$ respectively. All other representations may be obtained by taking tensor products of these. We will have use for the completely antisymmetric invariant tensors $\epsilon_{\alpha \beta \gamma \delta}$ and $\epsilon^{\alpha \beta \gamma \delta}$, defined so that $\epsilon_{\alpha \gamma \delta \epsilon} \epsilon^{\beta \gamma \delta \epsilon}=6 \delta_{\alpha}^{\beta}$. Another bosonic subgroup of $(2,0)$ supersymmetry is an $S O(5) R$-symmetry group. We denote its spinor representation, from which all other representations can be constructed, by an index $a, b, \ldots=1,2,3,4$. The anti-symmetric invariant tensors are denoted $\Omega^{a b}$ and $\Omega_{a b}$ defined so that $\Omega_{a b} \Omega^{b c}=\delta_{a}^{c}$.

The $(2,0)$ supersymmetry algebra has a well-known representation on a free field theory known as a tensor multiplet [4. We denote the bosonic fields as $b_{\alpha}^{\beta}$ and $\phi^{a b}$, subject to the algebraic constraints $b_{\alpha}^{\alpha}=0, \phi^{a b}=-\phi^{b a}$ and $\phi^{a b} \Omega_{a b}=0$. This means that $b_{\alpha}^{\beta}$ is a two-form, and that the scalars $\phi^{a b}$ transform in the vector representation of the $R$-symmetry group. These fields are real. (This means that the components $b_{\alpha}^{\beta}$ and $\phi^{a b}$ are related to the complex conjugates of other components by certain linear conditions.) The fermionic fields are denoted as $\psi_{\alpha}^{a}$, i.e. it is a chiral spinor in the spinor representation of the $R$-symmetry group. This field obeys a symplectic Majorana reality condition.

The field $b_{\alpha}^{\beta}$ is subject to a gauge-invariance with a one-form parameter $a_{\alpha \beta}=$ $-a_{\beta \alpha}$, which acts as $b_{\alpha}^{\beta} \rightarrow b_{\alpha}^{\beta}+\partial_{\alpha \gamma} a^{\gamma \beta}+\frac{1}{4} \delta_{\alpha}^{\beta} \partial_{\gamma \delta} a^{\gamma \delta}$. Here $\partial_{\alpha \beta}=-\partial_{\beta \alpha}$ is the ordinary derivative acting on the space-time coordinates $x^{\alpha \beta}=-x^{\beta \alpha}$. It is convenient to also introduce $\partial^{\alpha \beta}=\frac{1}{2} \epsilon^{\alpha \beta \gamma \delta} \partial_{\gamma \delta}$. The gauge invariant information of $b_{\alpha}^{\beta}$ is contained in the self-dual and anti self-dual three-form field strengths $h_{\alpha \beta}$ and $h^{\alpha \beta}$ defined as

$$
\begin{aligned}
h_{\alpha \beta} & =\partial_{\alpha \gamma} b_{\beta}^{\gamma}+\partial_{\beta \gamma} b_{\alpha}^{\gamma} \\
h^{\alpha \beta} & =\partial^{\alpha \gamma} b_{\gamma}^{\beta}+\partial^{\beta \gamma} b_{\gamma}^{\alpha} .
\end{aligned}
$$

They obey the Bianchi identity $\partial^{\alpha \gamma} h_{\alpha \beta}-\partial_{\alpha \beta} h^{\alpha \gamma}=0$.

The dynamics of the fields $b_{\alpha}^{\beta}, \phi^{a b}$ and $\psi_{\alpha}^{a}$ is described by the tensor multiplet action

$$
S_{T M}=\int_{M} d^{6} x\left(2 h_{\alpha \beta} h^{\alpha \beta}-\Omega_{a c} \Omega_{b d} \partial_{\alpha \beta} \phi^{a b} \partial^{\alpha \beta} \phi^{c d}-4 i \Omega_{a b} \psi_{\alpha}^{a} \partial^{\alpha \beta} \psi_{\beta}^{b}\right) .
$$


Under an arbitary variation of the fields, the variation of the action is

$$
\delta S_{T M}=\int_{M} d^{6} x\left(8 \delta b_{\alpha}^{\beta} \partial^{\alpha \gamma} h_{\beta \gamma}+2 \delta \phi^{a b} \Omega_{a c} \Omega_{b d} \partial^{\alpha \beta} \partial_{\alpha \beta} \phi^{c d}-8 i \delta \psi_{\alpha}^{a} \Omega_{a b} \partial^{\alpha \beta} \psi_{\beta}^{b}\right),
$$

from which the classical equations of motion can be read off:

$$
\begin{aligned}
\partial^{\alpha \gamma} h_{\beta \gamma} & =0 \\
\partial^{\alpha \beta} \partial_{\alpha \beta} \phi^{a b} & =0 \\
\partial^{\alpha \beta} \psi_{\beta}^{b} & =0
\end{aligned}
$$

Apart from its manifest Lorentz and $R$-symmetries, this action is also invariant under supersymmetries with constant infinitesimal fermionic parameters $\eta_{a}^{\alpha}$ acting as

$$
\begin{aligned}
\delta \phi^{a b} & =-i \eta_{c}^{\gamma}\left(\Omega^{c a} \psi_{\gamma}^{b}+\Omega^{b c} \psi_{\gamma}^{a}+\frac{1}{2} \Omega^{a b} \psi_{\gamma}^{c}\right) \\
\delta \psi_{\alpha}^{a} & =\eta_{c}^{\gamma}\left(\Omega^{a c} h_{\alpha \gamma}+2 \partial_{\alpha \gamma} \phi^{a c}\right) \\
\delta b_{\alpha}^{\beta} & =-i \eta_{a}^{\beta} \psi_{\alpha}^{a}+\frac{i}{4} \delta_{\alpha}^{\beta} \eta_{c}^{\gamma} \psi_{\gamma}^{c} .
\end{aligned}
$$

The transformation law for $b_{\alpha}^{\beta}$ implies that the field strengths $h_{\alpha \beta}$ and $h^{\alpha \beta}$ transform as

$$
\begin{aligned}
\delta h_{\alpha \beta} & =i \eta_{c}^{\gamma}\left(\partial_{\gamma \alpha} \psi_{\beta}^{c}+\partial_{\gamma \beta} \psi_{\alpha}^{c}\right) \\
\delta h^{\alpha \beta} & =-i \eta_{a}^{\alpha} \partial^{\beta \gamma} \psi_{\gamma}^{a}-i \eta_{a}^{\beta} \partial^{\alpha \gamma} \psi_{\gamma}^{a} .
\end{aligned}
$$

We note that the anti self-dual part $h^{\alpha \beta}$ of the field strength of $b_{\alpha}^{\beta}$ does not appear in the right hand sides of these supersymmetry transformation laws. Furthermore, the supersymmetry variation $\delta h^{\alpha \beta}$ vanishes when the equations of motion of the field $\psi_{\alpha}^{a}$ is imposed. These two properties imply that $h^{\alpha \beta}$ is not really part of the tensor multiplet, which thus consists solely of the fields $\phi^{a b}, \psi_{\alpha}^{a}$, and $h_{\alpha \beta}$. It is well-known that a covariant action describing only a tensor multiplet does not exist. The above action describes a free tensor multiplet and a free anti self-dual field strength $h^{\alpha \beta}$. Later in this paper, we will construct interaction terms that couple the tensor multiplet to a string. We will however make sure that $h^{\alpha \beta}$ remains decoupled from all other degrees of freedom.

The fields $\phi^{a b}$ and $\psi_{\alpha}^{a}$ take their values in linear spaces, so the numerical coefficients in their kinetic terms in $S_{T M}$ are purely conventional, and just reflects the normalization of these fields. But the field $b_{\alpha}^{\beta}$ is really a connection on a 1-gerbe, so its normalization cannot be changed. The constant in front of its kinetic term in $S_{T M}$ is therefore significant. A priori, it is a free parameter of the theory, but by considering the theory on a space-time of non-trivial topology, one finds that the decoupling of the anti self-dual part $h^{\alpha \beta}$ of the field strength is only consistent for a specific value of this constant. (In this paper, we will make no attempt to define our conventions precisely enough to determine this value.) So the action $S_{T M}$ is in fact unique, with no free parameters. 


\section{The on-shell superspace of a free tensor multiplet}

Although the aim of this paper is to construct an interacting theory, for which no superspace formulation is known, it is still useful to introduce the on-shell superspace of the free tensor multiplet described in the previous section [4.

Superspace is parametrized by the bosonic coordinates $x^{\alpha \beta}$ of six-dimensional Minkowski space together with a set of fermionic coordinates $\theta_{a}^{\alpha}$, which are symplectic Majorana. Supersymmetry transformations are generated by the supercharges

$$
Q_{\alpha}^{a}=\partial_{\alpha}^{a}-i \Omega^{a b} \theta_{b}^{\beta} \partial_{\alpha \beta},
$$

where $\partial_{\alpha}^{a}=\frac{\partial}{\partial \theta_{a}^{\alpha}}$. These anti-commute with the covariant derivatives

$$
D_{\alpha}^{a}=\partial_{\alpha}^{a}+i \Omega^{a b} \theta_{b}^{\beta} \partial_{\alpha \beta}
$$

We will also need differential forms on superspace. As a basis of one-forms, we will use $e^{\alpha \beta}=-e^{\beta \alpha}$ and $d \theta_{a}^{\alpha}$, where

$$
e^{\alpha \beta}=d x^{\alpha \beta}+\frac{i}{2} \Omega^{a b}\left(\theta_{a}^{\alpha} d \theta_{b}^{\beta}-\theta_{a}^{\beta} d \theta_{b}^{\alpha}\right) .
$$

These are dual to the tangent vectors $\partial_{\alpha \beta}$ and $D_{\alpha}^{a}$ respectively, so that the exterior derivative $d$ takes the form

$$
d=e^{\alpha \beta} \partial_{\alpha \beta}+d \theta_{a}^{\alpha} D_{\alpha}^{a}
$$

It follows that $d e^{\alpha \beta}=i \Omega^{a b} d \theta_{a}^{\alpha} \wedge d \theta_{b}^{\beta}$, whereas $d \theta$ of course is closed.

We now introduce a superfield $\Phi^{a b}(x, \theta)$, subject to algebraic constraints analogous to those of $\phi^{a b}(x)$, i.e. $\Phi^{a b}=-\Phi^{b a}$ and $\Omega_{a b} \Phi^{a b}=0$. It should also fulfill the differential constraint

$$
D_{\alpha}^{a} \Phi^{b c}+\frac{1}{5} \Omega_{d e} D_{\alpha}^{d}\left(2 \Omega^{a b} \Phi^{e c}-2 \Omega^{a c} \Phi^{e b}+\Omega^{b c} \Phi^{e a}\right)=0 .
$$

To analyse the implications of these constraints, it is convenient to define the superfields $\Psi_{\alpha}^{a}$ and $H_{\alpha \beta}$ as

$$
\begin{aligned}
\Psi_{\alpha}^{a} & =-\frac{2 i}{5} \Omega_{b c} D_{\alpha}^{b} \Phi^{c a} \\
H_{\alpha \beta} & =\frac{1}{4} \Omega_{a b} D_{\alpha}^{a} \Psi_{\beta}^{b} .
\end{aligned}
$$

It immediately follows that $H_{\alpha \beta}$ obeys the algebraic constraint $H_{\alpha \beta}=H_{\beta \alpha}$. Furthermore, the differential constraint on $\Phi^{a b}$ implies that all fermionic derivatives of $\Phi^{a b}$ may be expressed in terms of $\Phi^{a b}, \Psi_{\alpha}^{a}, H_{\alpha \beta}$ and bosonic derivatives thereof. Notably

$$
\begin{aligned}
D_{\alpha}^{a} \Phi^{b c} & =-i\left(\Omega^{a b} \Psi_{\alpha}^{c}+\Omega^{c a} \Psi_{\alpha}^{b}+\frac{1}{2} \Omega^{b c} \Psi_{\alpha}^{a}\right) \\
D_{\alpha}^{a} \Psi_{\beta}^{b} & =2 \partial_{\alpha \beta} \Phi^{a b}-\Omega^{a b} H_{\alpha \beta} \\
D_{\alpha}^{a} H_{\beta \gamma} & =i\left(\partial_{\alpha \beta} \Psi_{\gamma}^{a}+\partial_{\alpha \gamma} \Psi_{\beta}^{a}\right) .
\end{aligned}
$$


Finally, the differential constraint also implies the equations of motion

$$
\begin{aligned}
\partial^{\alpha \gamma} H_{\beta \gamma} & =0 \\
\partial^{\alpha \beta} \partial_{\alpha \beta} \Phi^{a b} & =0 \\
\partial^{\alpha \beta} \Psi_{\beta}^{b} & =0 .
\end{aligned}
$$

We will also need a certain three-form $F$ on superspace [7, 8] defined as

$F=-\frac{1}{12} e^{\alpha \beta} \wedge e^{\gamma \delta} \wedge e^{\epsilon \kappa} \epsilon_{\beta \gamma \delta \epsilon} H_{\alpha \kappa}-\frac{i}{4} e^{\alpha \beta} \wedge e^{\gamma \delta} \wedge d \theta_{a}^{\epsilon} \epsilon_{\beta \gamma \delta \epsilon} \Psi_{\alpha}^{a}+\frac{i}{4} e^{\alpha \beta} \wedge d \theta_{a}^{\gamma} \wedge d \theta_{b}^{\delta} \epsilon_{\alpha \beta \gamma \delta} \Phi^{a b}$

Acting with the exterior derivative $d$ on $F$, and using the above properties of the superfields $\Phi^{a b}, \Psi_{\alpha}^{a}$, and $H_{\alpha \beta}$, one finds that $F$ is closed, i.e. $d F=0$.

The general solution to the above constraints on the superfield $\Phi^{a b}(x, \theta)$ may be written in terms of component fields $\phi^{a b}(x), \psi_{\alpha}^{a}(x)$, and $h_{\alpha \beta}(x)$, that are the lowest components of the superfields $\Phi^{a b}(x, \theta), \Psi_{\alpha}^{a}(x, \theta)$, and $H_{\alpha \beta}(x, \theta)$ respectively. It follows that these component fields have to obey the algebraic constraints and the free classical equations of motion of the tensor multiplet fields discussed in the previous section. Including terms up to bilinear order in $\theta$, one finds that

$$
\begin{aligned}
\Phi^{a b}= & \phi^{a b}-i \theta_{c}^{\alpha}\left(\Omega^{c a} \psi_{\alpha}^{b}+\Omega^{b c} \psi_{\alpha}^{a}+\frac{1}{2} \Omega^{a b} \psi_{\alpha}^{c}\right) \\
& +i \theta_{c}^{\alpha} \theta_{d}^{\beta}\left(h_{\alpha \beta}\left(\Omega^{d a} \Omega^{b c}+\frac{1}{4} \Omega^{d c} \Omega^{a b}\right)-\Omega^{d a} \partial_{\alpha \beta} \phi^{b c}-\Omega^{d b} \partial_{\alpha \beta} \phi^{c a}\right)+\mathcal{O}\left(\theta^{3}\right) .
\end{aligned}
$$

The coefficients of the neglected terms are given by derivatives of the components fields $\phi^{a b}, \psi_{\alpha}^{a}$, and $h_{\alpha \beta}$, and the expansion ends with a term of order $\theta^{16}$. Acting with a supersymmetry transformation, generated by the supercharges, one finds that the transformation laws of the component fields agree with those presented in the previous section, thus completing their identification with the tensor multiplet fields.

\section{The Nambu-Goto and Wess-Zumino terms}

In this section, we will couple a string to a fixed tensor multiplet background, that fulfills the free equations of motion described in section two. This is an intermediate step, before constructing the complete dynamical theory of tensor multiplets and strings in the next section.

On physical grounds, we expect two types of couplings to appear: The first is a Nambu-Goto term given by an integral over the string world-sheet $\Sigma$. The integrand is the volume form on $\Sigma$, induced by its embedding into Minkowski space, times the $R$-symmetry invariant norm of the scalar fields $\phi^{a b}$. This term thus reflects the fact that the tension of the string is determined by the moduli. The second term is a Wess-Zumino term given by an integral over a Dirac membrane world-volume $D$, the boundary $\partial D$ of which equals the string world-sheet $\Sigma$. The integrand is the pullback of the self-dual field strength $h_{\alpha \beta}$ to $D$. This term thus encodes the electromagnetic 
coupling of the self-dual string to the field $b_{\alpha}^{\beta}$. Our problem is now to construct the supersymmetric versions of these couplings.

The embedding of the Dirac membrane world-volume $D$ in superspace is described by world-volume fields $X^{\alpha \beta}$ and $\Theta_{a}^{\alpha}$. They transform non-linearly under supersymmetry according to

$$
\begin{aligned}
\delta X^{\alpha \beta} & =\frac{i}{2} \Omega^{a b}\left(\eta_{a}^{\alpha} \Theta_{b}^{\beta}-\eta_{a}^{\beta} \Theta_{b}^{\alpha}\right) \\
\delta \Theta_{a}^{\alpha} & =-\eta_{a}^{\alpha} .
\end{aligned}
$$

It follows that the pullbacks to $D$ of the one-forms $e^{\alpha \beta}$ and $d \theta_{a}^{\alpha}$ introduced in the previous section, i.e. $E^{\alpha \beta}$ given by

$$
E^{\alpha \beta}=d X^{\alpha \beta}+\frac{i}{2} \Omega^{a b}\left(\Theta_{a}^{\alpha} d \Theta_{b}^{\beta}-\Theta_{a}^{\beta} d \Theta_{b}^{\alpha}\right)
$$

and $d \Theta_{a}^{\alpha}$, are invariant under supersymmetry. If we parametrize the string worldsheet $\Sigma$ with coordinates $\sigma^{i}, i=1,2$ and expand the one-forms $E^{\alpha \beta}$ in the basis $d \sigma^{i}$ as $E^{\alpha \beta}=d \sigma^{i} E_{i}^{\alpha \beta}$, we can construct a supersymmetric induced metric $G_{i j}$ on $\Sigma$ as

$$
G_{i j}=\frac{1}{4} \epsilon_{\alpha \beta \gamma \delta} E_{i}^{\alpha \beta} E_{j}^{\gamma \delta}
$$

We denote the determinant of this metric as $G$.

Supersymmetry transformations on the superfield $\Phi^{a b}$ are generated by the supercharges $Q_{\alpha}^{a}$. It follows that the pullback ${ }^{*} \Phi^{a b}=\Phi^{a b}(X, \Theta)$ of $\Phi^{a b}$ to $D$ is invariant under supersymmetry, since the contributions to its variation from the two terms in $Q_{\alpha}^{a}$ are precisely cancelled by the contributions due to the variations of its arguments $X$ and $\Theta$. It is then straightforward to construct a supersymmetric Nambu-Goto term $S_{N G}$ as

$$
S_{N G}=-\int_{\Sigma} d^{2} \sigma \sqrt{*(\Phi \cdot \Phi)} \sqrt{-G}
$$

where $\Phi \cdot \Phi$ is defined as

$$
\Phi \cdot \Phi=\frac{1}{4} \Omega_{a c} \Omega_{b d} \Phi^{a b} \Phi^{c d} .
$$

Similarly, the pullbacks of the superfields $\Psi_{\alpha}^{a}$ and $H_{\alpha \beta}$ to $D$ are invariant under supersymmetry, and so are the differentials $E^{\alpha \beta}$ and $d \Theta_{a}^{\alpha}$. We may thus construct a supersymmetric Wess-Zumino term $S_{W Z}$ as

$$
S_{W Z}=\int_{D}^{*} F,
$$

where ${ }^{*} F$ denotes the pullback to $D$ of the three-form $F$ introduced in the previous section.

The interaction terms must be invariant under a change of the Dirac membrane world-volume $D$, as long as its boundary is given by the string world-sheet $\Sigma$. An 
infinitesimal change of $D$ is parametrized by a superspace tangent vector field $\kappa$ on $D$. Expanding this as $\kappa=\kappa^{\alpha \beta} \partial_{\alpha \beta}+\kappa_{a}^{\alpha} D_{\alpha}^{a}$, we get the transformation laws

$$
\begin{aligned}
\delta X^{\alpha \beta} & =\kappa^{\alpha \beta}+\frac{i}{2} \Omega^{a b}\left(\kappa_{a}^{\alpha} \Theta_{b}^{\beta}-\kappa_{a}^{\beta} \Theta_{b}^{\alpha}\right) \\
\delta \Theta_{a}^{\alpha} & =\kappa_{a}^{\alpha},
\end{aligned}
$$

for the world-volume fields $X^{\alpha \beta}$ and $\Theta_{a}^{\alpha}$ that describe the embedding of $D$ in superspace. The superfield $\Phi^{a b}$ is of course invariant under this transformation. To describe a change which leaves the boundary $\Sigma$ fixed, $\kappa$ should vanish there. We will, however, be slightly more general and only require that the bosonic components $\kappa^{\alpha \beta}$ vanish on $\Sigma$, whereas the fermionic components $\kappa_{a}^{\alpha}$ on $\Sigma$ are subject to the constraint

$$
\Gamma_{\beta}^{\alpha} \kappa_{a}^{\beta}=\gamma_{a}^{b} \kappa_{b}^{\alpha}
$$

Here $\Gamma_{\beta}^{\alpha}$ and $\gamma_{a}^{b}$ are defined as

$$
\begin{aligned}
\Gamma_{\beta}^{\alpha} & =\frac{1}{2} \frac{1}{\sqrt{-G}} \epsilon^{i j} E_{i}^{\alpha \gamma} E_{j}^{\delta \epsilon} \epsilon_{\beta \gamma \delta \epsilon} \\
\gamma_{a}^{b} & =\frac{1}{\sqrt{*(\Phi \cdot \Phi)}} \Omega_{a c}{ }^{*} \Phi^{c b}
\end{aligned}
$$

and fulfill the identities $\Gamma_{\beta}^{\alpha} \Gamma_{\gamma}^{\beta}=\delta_{\gamma}^{\alpha}, \gamma_{a}{ }^{b} \gamma_{b}{ }^{c}=\delta_{a}^{c}, \Gamma_{\alpha}^{\alpha}=0$, and $\gamma_{a}^{a}=0$. The constraint on $\kappa_{a}^{\alpha}$ on $\Sigma$ thus means that it has eight linearly independent components, so that eight of the sixteen components of $\Theta_{a}^{\alpha}$ may be eliminated by a $\kappa$-symmetry transformation. The remaining eight components of $\Theta_{a}^{\alpha}$ correspond to four fermionic degrees of freedom. This equals the number of bosonic degrees of freedom, after eliminating two components of $X^{\alpha \beta}$ by invariance under reparametrizations of $\Sigma$. We remark that in many cases that have appeared in the literature, the integrand of the Wess-Zumino term is a total derivative, so by Stokes' theorem this term can be rewritten as an integral over the boundary $\Sigma$. The relevant part of the $\kappa$-symmetry is then of course parametrized by the fermionic components $\kappa_{a}^{\alpha}$ subject to the above constraint.

It remains to show that the sum of the interaction terms $S_{W Z}$ and $S_{N G}$ is indeed invariant under the transformation parametrized by the vector field $\kappa$ on $D$ described above. Let $\omega$ be an arbitary differential form on superspace, invariant under $\kappa$-symmetry. Its pullback $* \omega$ to $D$ then transforms as

$$
\delta^{*} \omega={ }^{*}\left(\iota_{\kappa} d \omega\right)+d^{*}\left(\iota_{\kappa} \omega\right)
$$

where $\iota_{\kappa}$ denotes contraction with the tangent vector $\kappa$. When this formula is applied to $\omega=F$, the first term vanishes since $F$ is closed. Stokes' theorem then gives the variation of the Wess-Zumino term as

$$
\delta S_{W Z}=\int_{\Sigma}^{*}\left(\iota_{\kappa} F\right)=\int_{\Sigma} \kappa_{a}^{\alpha} \frac{i}{4} \epsilon_{\alpha \beta \gamma \delta}{ }^{*}\left(e^{\beta \gamma} \wedge e^{\delta \epsilon} \Psi_{\epsilon}^{a}-2 e^{\beta \gamma} \wedge d \theta_{b}^{\delta} \Phi^{a b}\right),
$$


where we have used the vanishing of the bosonic components of $\kappa$ on $\Sigma$ in the last equality.

The variation of the Nambu-Goto term is given by

$$
\delta S_{N G}=-\int_{\Sigma} d^{2} \sigma\left\{\frac{\sqrt{-G}}{\sqrt{*(\Phi \cdot \Phi)}}\left({ }^{*} \Phi \cdot \delta^{*} \Phi\right)+\sqrt{*(\Phi \cdot \Phi)} \frac{\delta(-G)}{2 \sqrt{-G}}\right\} .
$$

Here the transformation of the pullback of $\Phi^{a b}$ is given by $\delta^{*} \Phi^{a b}={ }^{*}\left(\iota_{\kappa} d \Phi^{a b}\right)=$ $i \kappa_{c}^{\alpha *}\left(\Omega^{a c} \Psi_{\alpha}^{b}-\Omega^{b c} \Psi_{\alpha}^{a}-\frac{1}{2} \Omega^{a b} \Psi_{\alpha}^{c}\right)$, where we have expressed the fermionic derivatives of $\Phi^{a b}$ in terms of $\Psi_{\alpha}^{a}$ and used the vanishing of the bosonic components of $\kappa$. Finally, we should use the constraints on $\kappa_{\alpha}^{a}$ that are valid on $\Sigma$. One then easily finds that the first term in $\delta S_{W Z}$ cancels against the first term in $\delta S_{N G}$. A trickier computation shows that the second term in $\delta S_{W Z}$ cancels against the second term in $S_{N G}$. The sum $S_{N G}+S_{W Z}$ is thus invariant under $\kappa$-symmetry.

We have seen that the relative coefficient between the Nambu-Goto and WessZumino terms is determined by the requirement of $\kappa$-symmetry. But since the WessZumino term represents an electromagnetic coupling of the string to the field $b_{\alpha}^{\beta}$, its coefficient must obey Dirac quantization, i.e. it is given by an integer in appropriate units. We expect our strings to have the minimal non-trivial charge, which thus fixes this value completely. So there are no free parameters in the linear interaction terms.

\section{The Dirac-Dirac term}

In this section, we will describe how to construct the complete dynamical theory of an interacting tensor multiplet and a string. The main problem is as follows: We still define $\Phi^{a b}, \Psi_{\alpha}^{a}, H_{\alpha \beta}$, and $F$ in terms of the tensor multiplet fields $\phi^{a b}, \psi_{\alpha}^{a}$, and $h_{\alpha \beta}$ precisely as before using equations (16), (12), and (15). But crucial properties of these quantities are only valid when the tensor multiplet fields obey their free equations of motion (4), and thus cannot be used in the interacting theory. Indeed, for generic configurations of the tensor multiplet fields, these quantitites do not transform as superfields, so the interaction terms $S_{N G}$ and $S_{W Z}$ are not invariant under supersymmetry. Furthermore, the fermionic derivatives of $\Phi^{a b}$ are no longer related by the differential constraint (11), i.e. they cannot all be expressed in terms of $\Psi_{\alpha}^{a}$, and this invalidates the conclusion that $F$ is closed. Thus the sum $S_{N G}+S_{W Z}$ is not invariant under $\kappa$-symmetry. In this section we will show how these problems can be remedied by supplementing the tensor multiplet action $S_{T M}$ and the interaction terms $S_{N G}$ and $S_{W Z}$ with a direct Dirac membrane interaction $S_{D D}$. The transformation laws of the tensor multiplet fields under supersymmetry and $\kappa$-symmetry also have to be modified, by including terms supported on the Dirac membrane world volume $D$ and its boundary $\Sigma$. 


\subsection{Off-shell $\kappa$-symmetry}

The interaction $S_{N G}+S_{W Z}$ is invariant under a $\kappa$-symmetry transformation as described in the previous section, provided that the tensor multiplet fields $\phi^{a b}, \psi_{\alpha}^{a}$, and $h_{\alpha \beta}$ fulfill their free equations of motion. Its variation when no such on-shell constraints are imposed must therefore be of the form

$$
\delta\left(S_{N G}+S_{W Z}\right)=\int_{M} d^{6} x\left(-8 \check{b}_{\alpha}^{\beta} \partial^{\alpha \gamma} h_{\beta \gamma}-2 \check{\phi}^{a b} \Omega_{a c} \Omega_{b d} \partial^{\alpha \beta} \partial_{\alpha \beta} \phi^{c d}+8 i \check{\psi}_{\alpha}^{a} \Omega_{a b} \partial^{\alpha \beta} \psi_{\beta}^{b}\right),
$$

for some coefficient functions $\check{b}_{\alpha}^{\beta}, \check{\phi}^{a b}$, and $\check{\psi}_{\alpha}^{a}$ that are linear in the parameters $\kappa$ of the transformation. This variation can thus be cancelled by a variation $\delta_{1} S_{T M}$ of the tensor multiplet action, i.e.

$$
\delta\left(S_{N G}+S_{W Z}\right)+\delta_{1} S_{T M}=0,
$$

if we let the tensor multiplet fields transform as

$$
\begin{aligned}
\delta_{1} b_{\alpha}^{\beta} & =\check{b}_{\alpha}^{\beta} \\
\delta_{1} \phi^{a b} & =\check{\phi}^{a b} \\
\delta_{1} \psi_{\alpha}^{a} & =\check{\psi}_{\alpha}^{a} .
\end{aligned}
$$

But the latter transformations lead to an additional variation $\delta_{1}\left(S_{N G}+S_{W Z}\right)$ of the interaction. We hope to cancel this by adding an extra Dirac-Dirac term $S_{D D}$ to the action, constructed so that

$$
\delta_{1}\left(S_{N G}+S_{W Z}\right)+\delta S_{D D}=0 .
$$

If furthermore $S_{D D}$ is independent of the tensor multiplet fields, and only depends on the world volume fields $X^{\alpha \beta}$ and $\Theta_{a}^{\alpha}$, the transformation laws of which have not been altered, then $\delta_{1} S_{D D}=0$, and the complete action

$$
S=S_{T M}+S_{N G}+S_{W Z}+S_{D D}
$$

is exactly invariant under the modified $\kappa$-symmetry.

To see that such a term $S_{D D}$ indeed exists, we will have to retrace the above steps a bit more carefully. Repeating the analysis of the previous section but retaining also terms proportional to the free equations of motion of the tensor multiplet fields, we find that

$$
\delta\left(S_{N G}+S_{W Z}\right)=\int_{D}^{*}\left(\iota_{\kappa} d F\right)+\ldots
$$

In this and subsequent formulas, ... denotes terms that are supported on the string world-sheet $\Sigma$. The four-form $d F$ vanishes when the tensor multiplet fields fulfill their free equations of motion, so it can be written as

$$
\begin{aligned}
d F= & \int_{M} d^{6} x^{\prime}\left(-8 \tilde{b}_{\alpha}^{\beta}\left(x^{\prime}\right) \partial^{\prime \alpha \gamma} h_{\gamma \beta}\left(x^{\prime}\right)-2 \tilde{\phi}^{a b}\left(x^{\prime}\right) \partial^{\prime \alpha \beta} \partial_{\alpha \beta}^{\prime} \phi^{c d}\left(x^{\prime}\right) \Omega_{a c} \Omega_{b d}+\right. \\
& \left.+8 i \tilde{\psi}_{\alpha}^{a}\left(x^{\prime}\right) \partial^{\prime \alpha \beta} \psi_{\beta}^{b}\left(x^{\prime}\right) \Omega_{a b}\right)
\end{aligned}
$$


Here, $\tilde{b}_{\alpha}^{\beta}\left(x^{\prime}\right), \tilde{\psi}_{\alpha}^{a}\left(x^{\prime}\right)$, and $\tilde{\phi}^{a b}\left(x^{\prime}\right)$ are some four-forms on superspace with coordinates $x^{\alpha \beta}$ and $\theta_{a}^{\alpha}$, but as indicated they also depend on the variables $x^{\prime \alpha \beta}$. We note that they are closed (since $d F$ is closed), and independent of the tensor multiplet fields (since $d F$ is linear in these). The transformation laws of the tensor multiplet fields thus become

$$
\begin{aligned}
\delta_{1} \phi^{a b}\left(x^{\prime}\right) & =\int_{D}^{*}\left(\iota_{\kappa} \tilde{\phi}^{a b}\right)+\ldots \\
\delta_{1} \psi_{\alpha}^{a}\left(x^{\prime}\right) & =\int_{D}^{*}\left(\iota_{\kappa} \tilde{\psi}_{\alpha}^{a}\right)+\ldots \\
\delta_{1} h_{\alpha \beta}\left(x^{\prime}\right) & =\int_{D}^{*}\left(\iota_{\kappa} \tilde{h}_{\alpha \beta}\right)+\ldots
\end{aligned}
$$

where we have defined the closed four-form $\tilde{h}_{\alpha \beta}\left(x^{\prime}\right)$ as

$$
\tilde{h}_{\alpha \beta}\left(x^{\prime}\right)=\partial_{\alpha \gamma}^{\prime} \tilde{b}_{\beta}^{\gamma}+\partial_{\beta \gamma}^{\prime} \tilde{b}_{\alpha}^{\gamma} .
$$

Since the cohomology of superspace is trivial, the closed forms $\tilde{\phi}^{a b}\left(x^{\prime}\right), \tilde{\psi}_{\alpha}^{a}\left(x^{\prime}\right)$, and $\tilde{h}_{\alpha \beta}\left(x^{\prime}\right)$ are in fact exact and can thus be written as

$$
\begin{aligned}
\tilde{\phi}^{a b}\left(x^{\prime}\right) & =d \hat{\phi}^{a b}\left(x^{\prime}\right) \\
\tilde{\psi}_{\alpha}^{a}\left(x^{\prime}\right) & =d \hat{\psi}_{\alpha}^{a}\left(x^{\prime}\right) \\
\tilde{h}_{\alpha \beta}\left(x^{\prime}\right) & =d \hat{h}_{\alpha \beta}\left(x^{\prime}\right)
\end{aligned}
$$

with some three-forms $\hat{\phi}^{a b}\left(x^{\prime}\right), \hat{\psi}_{\alpha}^{a}\left(x^{\prime}\right)$, and $\hat{h}_{\alpha \beta}\left(x^{\prime}\right)$ that are functionals of the worldvolume fields $X^{\alpha \beta}$ and $\Theta_{a}^{\alpha}$. The exterior derivative $d$ on the right hand side of course acts on the superspace coordinates $x^{\alpha \beta}$ and $\theta_{a}^{\alpha}$. (The closed form $\tilde{b}_{\alpha}^{\beta}\left(x^{\prime}\right)$ is also exact, but we cannot express it as the exterior derivative $d$ acting on some local functional of $X^{\alpha \beta}$ and $\Theta_{a}^{\alpha}$.) We can now use the $\kappa$-symmetry transformation law (26) for the pullback of a differential form applied to the three-forms $\hat{\phi}^{a b}\left(x^{\prime}\right), \hat{\psi}_{\alpha}^{a}\left(x^{\prime}\right)$, and $\hat{h}_{\alpha \beta}\left(x^{\prime}\right)$. We thus find that the $\kappa$-symmetry transformations of the tensor multiplet fields are given by

$$
\begin{aligned}
\delta_{1} \phi^{a b}\left(x^{\prime}\right) & =\delta \int_{D}^{*} \hat{\phi}^{a b}\left(x^{\prime}\right)+\ldots \\
\delta_{1} \psi_{\alpha}^{a}\left(x^{\prime}\right) & =\delta \int_{D}^{*} \hat{\psi}_{\alpha}^{a}\left(x^{\prime}\right)+\ldots \\
\delta_{1} h_{\alpha \beta}\left(x^{\prime}\right) & =\delta \int_{D}^{*} \hat{h}_{\alpha \beta}\left(x^{\prime}\right)+\ldots
\end{aligned}
$$

where $\delta$ on the right hand sides denotes the $\kappa$-symmetry variation acting on the worldvolume fields $X^{\alpha \beta}$ and $\Theta_{a}^{\alpha}$. Again, the ... denote terms that are supported on the boundary $\Sigma$. For $\Phi^{a b}\left(x^{\prime}, \theta^{\prime}\right)$, we thus get the transformation law

$$
\delta_{1} \Phi^{a b}\left(x^{\prime}, \theta^{\prime}\right)=\delta \int_{D}^{*} \hat{\Phi}^{a b}\left(x^{\prime}, \theta^{\prime}\right)+\ldots
$$


where the three-form $\hat{\Phi}^{a b}\left(x^{\prime}, \theta^{\prime}\right)$ is constructed out of $\hat{\phi}^{a b}\left(x^{\prime}\right), \hat{\psi}_{\alpha}^{a}\left(x^{\prime}\right)$ and $\hat{h}_{\alpha \beta}\left(x^{\prime}\right)$ in the same way as $\Phi^{a b}$ is constructed out of $\phi^{a b}, \psi_{\alpha}^{a}$, and $h_{\alpha \beta}$. The quantities $\Psi^{a b}\left(x^{\prime}, \theta^{\prime}\right)$ and $H_{\alpha \beta}\left(x^{\prime}, \theta^{\prime}\right)$ transform analogously. Finally, we consider the three-form $F^{\prime}$ in the variables $x^{\prime \alpha \beta}$ and $\theta_{a}^{\prime \alpha}$ obtained as the pullback of $F$ by the map $x^{\prime \alpha \beta} \mapsto x^{\alpha \beta}, \theta_{a}^{\prime \alpha} \mapsto \theta_{a}^{\alpha}$. It transforms as

$$
\delta_{1} F^{\prime}=\delta \int_{D}^{*} \hat{F}^{\prime}+\ldots
$$

Here, $\hat{F}^{\prime}$ is a three-form in $x^{\alpha \beta}$ and $\theta_{a}^{\prime \alpha}$ constructed out of $\hat{\Phi}^{a b}, \hat{\Psi}_{\alpha}^{a}$ and $\hat{H}_{\alpha \beta}$ in the same way as $F$ is constructed out of $\Phi^{a b}, \Psi_{\alpha}^{a}$ and $H_{\alpha \beta}$. It is thus also a three-form in the variables $x^{\alpha \beta}$ and $\theta_{a}^{\alpha}$, on which the pullback map * to $D$ acts.

We should now really compute the $\delta_{1}$ variation of $S_{N G}+S_{W Z}$. However, $S_{W Z}$ and $S_{N G}$ are given by integrals over $D$ and its boundary $\Sigma$ respectively, and the $\delta_{1}$ variations of the tensor multiplet fields is a sum of terms supported on $D$ and terms supported on $\Sigma$. We thus get a divergent expression. To regularize this, we introduce another open three-manifold $D^{\prime}$ with boundary $\Sigma^{\prime}$ by slightly perturbing $D$. We can now compute the $\delta_{1}$ variation of the regularized interaction terms $S_{N G}^{\prime}+S_{W Z}^{\prime}$ obtained by replacing $D$ and $\Sigma$ with $D^{\prime}$ and $\Sigma^{\prime}$ in $S_{N G}$ and $S_{W Z}$. For a generic perturbation, the intersection of $D^{\prime}$ with $\Sigma$ and the intersection of $\Sigma^{\prime}$ with $D$ are empty, while $D$ and $D^{\prime}$ intersect in isolated points. This is analogous to the Dirac veto familiar from the theory of magnetic monopoles in four space-time dimensions, where the Dirac string should be chosen so that it does not go through any particle, and furthermore the effects on a particle of electromagnetic fields caused by that particle itself are not considered. It is also analogous to the definition of the self-intersection number of a submanifold.

These properties of $D^{\prime}$ and $\Sigma^{\prime}$ mean that $\delta_{1} S_{N G}^{\prime}$ vanishes, whereas terms in $\delta_{1} F^{\prime}$ that are supported on $\Sigma$ can be neglected in the computation of $\delta_{1} S_{W Z}^{\prime}$. Using the results of the previous paragraph, we thus find that

$$
\delta_{1}\left(S_{N G}^{\prime}+S_{W Z}^{\prime}\right)+\delta S_{D^{\prime} D}=0,
$$

if we define the regularization $S_{D^{\prime} D}$ of the new interaction term $S_{D D}$ as

$$
S_{D^{\prime} D}=-\frac{1}{2} \int_{D^{\prime}} \int_{D}^{* * \prime} \hat{F}^{\prime}
$$

Here, ${ }^{* \prime}$ denotes the pullback to $D^{\prime}$ acting on the variables $x^{\prime \alpha \beta}$ and $\theta_{a}^{\prime \alpha}$. The factor $\frac{1}{2}$ arises because the variation $\delta$ acts not only on the fields $X^{\alpha \beta}$ and $\Theta_{a}^{\alpha}$ on $D$, but also on the fields $X^{\prime \alpha \beta}$ and $\Theta_{a}^{\prime \alpha}$ on $D^{\prime}$, which transform analogously. We see that $S_{D^{\prime} D}$ represents a direct Dirac membrane interaction. As required, $S_{D D}$ is independent of the tensor multiplet fields. This is ultimately a consequence of the fact that the Wess-Zumino term is not only of first order in the tensor multiplet fields, like the Nambu-Goto term, but furthermore linear in them. This completes the proof of $\kappa$ symmetry of the complete action $S$. 


\subsection{The bosonic theory}

There is no simple closed expression for the form $\hat{F}^{\prime}$ that appears in $S_{D D}$, but it can be constructed order by order as a polynomial in the fermionic variables $\theta_{a}^{\alpha}$ and $\theta_{a}^{\prime \alpha}$, just like for example the superfield $\Phi^{a b}$ is expanded in $\theta_{a}^{\alpha}$ in (16). It is instructive, though, to show the result of this procedure to lowest order in fermions, i.e. to determine the bosonic part $S^{\text {bos }}$ of the complete action $S$.

For this purpose, it is convenient to employ an index-free notation: The bosonic world-volume fields are thus a map $X$ from $D$ to space-time $M$. The bosonic spacetime fields are a two-form $b$ and an $R$-symmetry vector $\phi$ of scalars. The field strength $h=d b$ can be decomposed as $h=h_{+}+h_{-}$, where $h_{+}=\frac{1}{2}(h+* h)$ and $h_{-}=\frac{1}{2}(h-* h)$ are the self-dual and anti self-dual parts respectively. (The $*$ is the Hodge duality operator.) The bosonic parts of $S_{T M}, S_{N G}$, and $S_{W Z}$ are

$$
\begin{aligned}
S_{T M}^{\mathrm{bos}} & =\frac{1}{2} \int_{M} h \wedge * h+\frac{1}{2} \int_{M} d \phi \cdot \wedge * d \phi \\
S_{N G}^{\mathrm{bos}} & =\int_{\Sigma} \sqrt{\phi \cdot \phi} \sqrt{-g} \\
S_{W Z}^{\mathrm{bos}} & =\int_{D} 2 h_{+} .
\end{aligned}
$$

(In this subsection, to avoid confusion with the Hodge duality operator $*$, we suppress the ${ }^{*}$ that indicates the pullback of a differential form to $D$ or $\Sigma$.) Here $g$ denotes the determinant of the metric on $\Sigma$ induced by its embedding in six-dimensional Minkowski space. To proceed further, we need to introduce the Poincaré dual threeform $\delta_{D}$ of the three-manifold $D$ embedded in six-dimensional Minkowski space, defined by the property that

$$
\int_{M} \delta_{D} \wedge s=\int_{D} s
$$

for an arbitrary three-form $s$ on Minkowski space. It can be written as

$$
\delta_{D}=\left\langle d x \wedge d x \wedge d x \int_{D} d X \wedge d X \wedge d X\right\rangle \delta^{(6)}(x-X),
$$

where $\langle\ldots \ldots\rangle$ means that the three space-time differentials $d x$ and the three pullback differentials $d X$ are contracted with the six-dimensional Levi-Civita tensor. We can now write the bosonic part of $S_{D D}$ as

$$
S_{D D}^{\mathrm{bos}}=\frac{1}{2} \int_{D} * \delta_{D}
$$

As described in the previous subsection, this expression is divergent since $* \delta D$ is supported on $D$, and has to be regularized by replacing one copy of $D$ with a perturbation $D^{\prime}$. The complete bosonic action $S^{\text {bos }}=S_{T M}^{\text {bos }}+S_{N G}^{\text {bos }}+S_{W Z}^{\text {bos }}+S_{D D}^{\text {bos }}$ can be rewritten as

$$
S^{\mathrm{bos}}=\frac{1}{2} \int_{M} h^{\mathrm{tot}} \wedge * h^{\mathrm{tot}}+\int_{M} d \phi \cdot \wedge * d \phi+\int_{\Sigma} \sqrt{\phi \cdot \phi} \sqrt{-g}+\int_{\Sigma} b
$$


where the total field strength $h^{\text {tot }}$ is defined as

$$
h^{\text {tot }}=h+\delta_{D} .
$$

The last term in the action is obtained by applying Stokes' theorem to the term $\int_{D} h$ and represents the standard electric coupling of the string to the field $b$. The first term can then be understood as a way of incorporating the magnetic coupling. Indeed, $h^{\text {tot }}$ fulfills a modified Bianchi identity $d h^{\text {tot }}=\delta_{\Sigma}$, where $\delta_{\Sigma}=d \delta_{D}$ is the Poincaré dual of the string world-sheet $\Sigma$. This is appropriate in the presence of a magnetically charged string.

An infinitesimal bosonic $\kappa$-symmetry transformation is parametrized by a vector $\kappa$ on $D$, subject to the condition that it vanishes on $\Sigma$. It acts by displacing $D$ while keeping the boundary $\Sigma$ fixed. The transformation law for $X$ is given by

$$
X \rightarrow X+\kappa
$$

It follows that the Poincaré dual $\delta_{D}$ changes by an exact form, $\delta_{D} \rightarrow \delta_{D}+d \lambda$, where the two-form $\lambda$ is given by

$$
\lambda=\left\langle d x \wedge d x \int_{D} \kappa d X \wedge d X \wedge d X\right\rangle \delta^{(6)}(x-X) .
$$

(The notation $\langle\ldots \ldots\rangle$ here means that the two space-time differentials $d x$, the vector $\kappa$, and the three pullback differentials $d X$ are contracted with the Levi-Civita tensor.) To arrive at this expression, we have used Stokes' theorem together with the condition that $\kappa$ vanishes on the boundary $\Sigma$ of $D$. We see that $h^{\text {tot }}$ is invariant under $\kappa$ symmetry, provided that the let the two-form $b$ transform according to

$$
b \rightarrow b-\lambda \text {. }
$$

Finally, the last term in $S^{\text {bos }}$ is invariant since the pullback of $\lambda$ to $\Sigma$ vanishes. This completes the proof of $\kappa$-symmetry for the bosonic action $S^{\text {bos }}$.

\subsection{Off-shell supersymmetry}

We now return to the complete theory including fermions. Off-shell supersymmetry is largely analogous to off-shell $\kappa$-symmetry: The statement that $\Phi^{a b}$ and $F$ transform as superfields is only valid provided that the tensor multiplet fields $\phi^{a b}, \psi_{\alpha}^{a}$, and $h_{\alpha \beta}$ fulfill their free equations of motion (4). It follows that the variation of the interaction $S_{N G}+S_{W Z}$ when no such constraints are imposed is a linear combination of $\partial^{\alpha \beta} \partial_{\alpha \beta} \phi, \partial^{\alpha \beta} \psi_{\beta}^{a}$, and $\partial^{\alpha \gamma} h_{\beta \gamma}$. It can thus be cancelled by supplementing the previous supersymmetry transformation laws of the tensor multiplet fields (5) with additional $\delta_{1}$ terms, chosen so that

$$
\delta\left(S_{N G}+S_{W Z}\right)+\delta_{1} S_{T M}=0 .
$$


But this leads to an additional variation $\delta_{1}\left(S_{N G}+S_{W Z}\right)$ of the interaction. We also need to take the variation $\delta S_{D D}$ of the additional term $S_{D D}$, which is independent of the tensor multiplet fields, into account.

The issue of divergences is precisely analogous to the case of $\kappa$-symmetry: The $\delta_{1}$ transformations contain terms supported on $D$ and on $\Sigma$, and $S_{N G}$ and $S_{W Z}$ are given by integrals over $\Sigma$ and $D$ respectively. We regulate the ensuing divergences by instead considering the $\delta_{1}$ variation of $S_{N G}^{\prime}$ and $S_{W Z}^{\prime}$ obtained by using a perturbed world-volume $D^{\prime}$ with boundary $\Sigma^{\prime}$. Also, $S_{D D}$ is regularized to $S_{D^{\prime} D}$. It follows from the properties of a generic perturbation $D^{\prime}$ that $\delta_{1} S_{N G}^{\prime}$ vanishes, so the total variation of the regularized action is

$$
\left(\delta+\delta_{1}\right) S=\delta_{1} S_{W Z}^{\prime}+\delta S_{D^{\prime} D},
$$

where furthermore terms in the $\delta_{1}$ variations of the tensor multiplet fields that are supported on $\Sigma$ may be neglected in the computation of $\delta_{1} S_{W Z}^{\prime}$. So the right hand side is given by a double integral over $D$ and $D^{\prime}$.

A direct proof of the vanishing of the last expression can probably be found, and is possibly quite illuminating. But in this paper we will content ourselves with a more implicit argument: We have shown in the beginning of this section that the complete action $S$ is invariant under the $\kappa$-transformations, by which $D$ may be arbitrarily chosen as long as its boundary is given by the string world-sheet $\Sigma$. But according to the last equation, the supersymmetry variation of $S$ is given by a double integral over two copies of $D$ (one of which is actually perturbed to $D^{\prime}$ ). We claim that the integrand of this expression must actually be exact either on $D$ or on $D^{\prime}$, so that the integral vanishes by Stokes' theorem. (The boundary term vanishes, since the intersection of $D$ with $\Sigma^{\prime}$ or vice versa is empty.) The reason is that any non-exact terms would mean that the supersymmetry variation of $S$ had a dependence on the choice of $D$, which contradicts the fact that $S$ itself is independent of such a choice. This completes the proof of supersymmetry of the complete action $S$.

To summarize, the complete action is

$$
\begin{aligned}
S= & \int_{M} d^{6} x\left(2 h_{\alpha \beta} h^{\alpha \beta}-\Omega_{a c} \Omega_{b d} \partial_{\alpha \beta} \phi^{a b} \partial^{\alpha \beta} \phi^{c d}-4 i \Omega_{a b} \psi_{\alpha}^{a} \partial^{\alpha \beta} \psi_{\beta}^{b}\right) \\
& -\int_{\Sigma} d^{2} \sigma \sqrt{{ }^{*}(\Phi \cdot \Phi)} \sqrt{-G}+\int_{D}{ }^{*} F-\frac{1}{2} \int_{D^{\prime}} \int_{D}{ }^{* * \prime} \hat{F}^{\prime} .
\end{aligned}
$$

We have shown that it is invariant under a local $\kappa$-symmetry, by which the Dirac membrane world-volume $D$ may be arbitarily chosen as long as its boundary is given by the string world-sheet $\Sigma$, and half of the components of $\Theta_{a}^{\alpha}$ on $\Sigma$ may be eliminated. This transformation acts on the string degrees of freedom in a standard way, but also on tensor multiplet fields with terms supported on $D$. Finally, we have shown that the action is invariant under global $(2,0)$ supersymmetry transformations, acting on the string degrees of freedom in the standard way, but with the standard transformation laws of the tensor multiplet fields supplemented with terms supported on $D$ and on $\Sigma$.

M.H. is a Research Fellow at the Royal Swedish Academy of Sciences. 


\section{References}

[1] E. Witten, Some comments on string dynamics, hep-th/9507121.

[2] P. Arvidsson, E. Flink and M. Henningson, Thomson scattering of chiral tensors and scalars against a self-dual string, JHEP 12 (2002) 010, hep-th/0210223.

P. Arvidsson, E. Flink and M. Henningson, Free tensor multiplets and strings in spontaneously broken six-dimensional $(2,0)$ theory, JHEP 06 (2003) 039, hep-th/0306145

P. Arvidsson, E. Flink and M. Henningson, Supersymmetric coupling of a self-dual string to a (2,0) tensor multiplet background, JHEP 11 (2003) 015, hep-th/0309244.

[3] E. Witten, Five-brane effective action in M-theory, J. Geom. Phys. 22 (1997) 103, hep-th/9610234.

[4] P.S. Howe, G. Sierra and P.K. Townsend, Supersymmetry in six dimensions, Nucl. Phys. B221 (1983) 331.

[5] S. Deser, A. Gomberoff, M. Henneaux and C. Teitelboim, p-brane dyons and electric-magnetic duality, Nucl. Phys. B520 (1998) 179, hep-th/9712189.

[6] E. Bergshoeff, E. Sezgin and P.K. Townsend, Supermembranes and elevendimensional supergravity, Phys. Lett. B189 (1987) 75.

[7] C. Grojean and J. Mourad, Superconformal $6 D(2,0)$ theories in superspace, Class. Quant. Grav. 15 (1998) 3397, hep-th/9807055.

[8] P.S. Howe, Aspects of the D =6, (2,0) tensor multiplet, Phys. Lett. B503 (2001) 197, hep-th/0008048. 